Published in final edited form as:

J Infect. 2008 September ; 57(3): 204-213. doi:10.1016/j.jinf.2008.06.017.

\title{
Transmission of Streptococcus pneumoniae in an Urban Slum Community
}

\author{
Joice Neves Reis ${ }^{1,2}$, Tania Palma ${ }^{1}$, Guilherme S. Ribeiro ${ }^{1}$, Ricardo M Pinheiro ${ }^{1}$, Cassio \\ Tâmara Ribeiro ${ }^{1}$, Soraia Machado Cordeiro ${ }^{1}$, H. P. da Silva Filho ${ }^{1}$, Monica Moschioni ${ }^{3}$, Terry \\ A. Thompson ${ }^{4}$, Brian Spratt ${ }^{5}$, Lee W. Riley ${ }^{6}$, Michele A. Barocchi ${ }^{3}$, Mitermayer G. Reis ${ }^{1}$, and \\ Albert I. Ko ${ }^{1,7}$ \\ 1Instituto Gonçalo Moniz, Fundação Oswaldo Cruz, Ministério da Saúde, Salvador, Brazil \\ 2Faculdade de Farmácia, Universidade Federal da Bahia, Salvador, Brazil
}

3Novartis Vaccines, Siena 53100, Italy

4Streptococcus Laboratory, Centers for Disease Control and Prevention, Atlanta, GA 30333

5Department of Infectious Disease Epidemiology, Imperial College London, London W2 1PG, UK

6Division of Infectious Diseases, School of Public Health, University of California, Berkeley, California 94720

7Division of International Medicine and Infectious Diseases, Department of Medicine, Weill Medical College of Cornell University, New York, NY 10021

\begin{abstract}
Background-Inhabitants of slum settlements represent a significant proportion of the population at risk for pneumococcal disease in developing countries.
\end{abstract}

Methods-We conducted a household survey of pneumococcal carriage among residents of a slum community in the city of Salvador, Brazil.

Results-Among 262 subjects, 95 (36\%) were colonized with S. pneumoniae. Children <5 years of age (OR, 8.0; 95\%CI, 3.5-18.6) and those who attended schools (OR 2.7, 95\%CI, 1.2-6.0) had significantly higher risk of being colonized. Of 94 isolates obtained from colonized individuals, $51 \%$ had serotypes included in the seven-valent pneumococcal conjugate vaccine. Overall, 10\% (9 of 94 isolates) were nonsusceptible to penicillin and $28 \%$ ( 27 of 94 isolates) were resistant to cotrimoxazole. BOX-PCR, PFGE and MLST analysis found that 44\% of the carriage isolates belonged to 14 distinct clonal groups. Strains of the same clonal group were isolated from multiple members of 9 out of the 39 study households. Nineteen carriage isolates had genotypes that were the same as those identified among 362 strains obtained from active surveillance for meningitis.

\section{Correspondence to: Joice Neves Reis.}

Reprints or correspondence: Joice Neves Reis, Ph.D Centro de Pesquisas Gonçalo Moniz, Fundação Oswaldo Cruz/MS Rua Waldemar Falcão, 121 Salvador, Bahia 40296-710; Brazil Telephone: (55 71) 3176-2302. Fax: (55 71) 3176-2281 e-mail: joice@bahia.fiocruz.br. Conflict of interest: The authors do not have any commercial or other association that might pose a conflict of interest with respect to the study, except for M.M. and M.A.B who are employees of Novartis Vaccines.

This work has not been presented previously in a meeting.

Publisher's Disclaimer: This is a PDF file of an unedited manuscript that has been accepted for publication. As a service to our customers we are providing this early version of the manuscript. The manuscript will undergo copyediting, typesetting, and review of the resulting proof before it is published in its final citable form. Please note that during the production process errorsmaybe discovered which could affect the content, and all legal disclaimers that apply to the journal pertain. 
Conclusions-The study's findings indicate that there is significant intra and inter-household spread of S. pneumoniae in the slum community setting. However, a limited number of clones encountered during carriage among slum residents were found to cause invasive disease.

\section{Keywords}

Streptococcus pneumoniae; nasopharyngeal carriage; pneumococcal conjugate vaccines; antibiotic resistance; urban slums

\section{INTRODUCTION}

Streptococcus pneumoniae is a major cause of severe infections such as meningitis, septicemia and pneumonia worldwide. Risk groups for invasive pneumococcal disease are young children under the age of 2 years, elderly people, and immunocompromised patients [1].

Nasopharyngeal carriage of pneumococci is likely to occur in all individuals at least once during infancy [2]. Infants are an important reservoir for community-wide transmission, which for the most part produces asymptomatic carriage [3]. Invasive pneumococcal disease is a relatively rare outcome and originates from colonization with a homologous strain [4]. Although a genetically-diverse population of pneumococcal clones are found in carriage, a limited number of clones appear to produce invasive disease [5,6]. To distinguish between clones that are capable of producing carriage or invasive disease, both clinical and nasopharyngeal isolates from the same geographic region and time period need to be characterized.

Several studies have compared carriage and invasive disease isolates from the same epidemiological setting [5-8]. A study performed in Oxford, England found that clones with the same serotype had similar invasive potential indicating that serotype may be more important than genotype in the ability of pneumococci to cause invasive disease [7]. In contrast, a study from Sweden identified clones that belonged to the same serotype but had different abilities to cause invasive disease. In addition, this study found that clones with different serotypes, which were presumably generated due capsular switch phenomenon, had the same disease potential [8].

At present, 1 billion of the world's population resides in urban slums, most of which are situated in developing countries [9]. Much of this marginalized population has not benefited from the advances in prevention afforded by immunization with pneumococcal protein-capsular conjugate vaccines [10]. Although underlying conditions of poverty presumably lead to high rates of pneumococci carriage and invasive disease, little information is available with respect to disease burden and transmission of S. pneumoniae within these communities. Salvador is the third largest city in Brazil (population, 2.8 million inhabitants) in which more than $60 \%$ of the population resides in slum settlements. Active surveillance in Salvador found that the annual incidence for pneumococcal meningitis was 31.7 and 8 cases per 100,000 person-years in children under one and five years of age, respectively [11]. The large majority (>80\%) of meningitis cases in the city were residents of slum communities (favelas). Children $<2$ years had a significantly increased risk of acquiring penicillin-nonsusceptible (PNSP) isolates than children $>2$ years. Furthermore, $50 \%$ of all the PNSP meningitis cases were caused by a single serotype 14 clone, which has disseminated throughout Brazil [11,12]. We herein report the findings of a cross-sectional study conducted in a slum community in Salvador, where ongoing surveillance for pneumococcal meningitis has been conducted, to identify the prevalence and risk factors for $S$. pneumoniae nasopharyngeal carriage among community residents. Furthermore, genotyping methods were used to determine the contribution of clones in the transmission of S. pneumoniae within households and the community. 


\section{METHODS}

\section{Study site and population}

The survey was carried out in the slum community (favela) of Nordeste de Amaralina in the city of Salvador, Brazil between July 2000 and May 2001. According to the national census bureau [13], 60\% of the 2.6 million inhabitants of Salvador resides in slums such as Nordeste de Amaralina. In 2000, the study community had a population of 23,980 inhabitants, distributed within 18 census district in an area of $0.5 \mathrm{~km}^{2}$ [14]. A representative census district was selected as the study within this community. We randomly selected 39 (13\%) of the 296 households (1,338 inhabitants) which were identified during a census of the study site. The study team performed house visits to identify eligible subjects, who were defined as residents living continuously in the selected household during the month prior to recruitment. Subjects were enrolled into the study according to written informed consent procedures approved by the Oswaldo Cruz Foundation, Brazilian Ministry of Health.

\section{Data collection}

Interviews were performed during the house visits to obtain information on demographics, underlying medical conditions, hospitalizations, occurrence of an upper respiratory tract infection (URTI) in the previous month, antibiotic therapy in the last month, childcare arrangements, school attendance and habits such as smoking. Information for children was obtained by interviewing the parent or legal guardian.

\section{Isolation of pneumococci}

Nasopharyngeal specimens were collected with calcium alginate swabs (Calgiswab type 1, Spectrum USA) and immediately streaked onto agar plates with $5 \%$ sheep blood and $5.0 \mu \mathrm{g} /$ $\mathrm{mL}$ of gentamicin. Plates were incubated at $35^{\circ} \mathrm{C}$ in $5 \% \mathrm{CO}_{2}$-enriched atmosphere for up to 48 hours. Three to five colonies exhibiting morphologic characteristics of $S$. pneumoniae were isolated. Pneumococci were identified according to Gram-stain morphology, optochin sensitivity and bile solubility.

\section{Antimicrobial susceptibility testing}

The disk-diffusion method was performed according to CLSI recommendations [15] to determine susceptibility of isolates to oxacillin, cefotaxime, tetracycline, erythromycin, clindamycin, cotrimoxazole, vancomycin, and chloramphenicol (BBL Microbiology Systems, Cockeysville, USA). Isolates exhibiting an inhibition zone diameter $<20 \mathrm{~mm}$ surrounding a $1 \mu \mathrm{g}$ oxacillin disk were tested for penicillin MIC by the E-test (AB Biodisk, Solna, Sweden). Isolates with a penicillin MIC value $\geq 0.1 \mu \mathrm{g} / \mathrm{ml}$ were defined as penicillin-nonsusceptible.

\section{Serotyping}

The Quellung reaction was performed to determine the serotype of pneumococcal isolates. For each study subject isolate, three to five colonies were picked from culture isolation plates and serotyped.

\section{Box Fingerprinting}

The Box polymerase chain reaction (Box-PCR) typing method was performed as described by Ko et al. [11]. The Gel Compar software version 4 (Applied Maths, Kortrijk, Belgium) was used to analyze digitalized images of gel electrophoresis patterns. The gel bands were assigned manually, according to analysis of densitometry curves and visual comparison with the accompanying copy of photograph. Strains that differed by $\leq 3$ bands were defined as being related and assigned the same letter code. Within a group of related patterns, distinct, non 
identical patterns were assigned a numerical index. An isolate was defined to have a cluster pattern if it had an identical gel pattern to one or more other isolates in the survey.

\section{Pulse-field gel electrophoresis (PFGE)}

PFGE analysis was performed to confirm the genotypes assigned in the BOX-PCR analysis. Chromosomal DNA fragments generated by SmaI digestion were prepared and analyzed as described elsewhere [16]. A CHEF-DRII apparatus (Bio-Rad, Hercules, CA) was used for running the gels. Interpretation of relatedness of isolates was performed according to the Tenover criteria [17].

\section{Multilocus sequence typing (MLST)}

MLST was performed, according to the procedure of Enright et al. [18], for all isolates which had cluster patterns by Box fingerprinting and a possibly-related pattern by PFGE and for a randomly selected group of 10 isolates with unrelated PFGE patterns. The Applied Biosystems Prism 3700 automated sequencer (PE Applied Biosystems) was used to sequence housekeeping genes ( $a r o E$, $g d h, g k i, r e c P$, spi, xpt and $d d l$ ) from chromosomal DNA obtained from pneumococcal isolates. Sequences were submitted to the online MLST database (http://www.mlst.net), which in turn assigned alleles at each locus and a sequence type.

\section{Data analysis}

Epi-Info Version 6.04 software system (Center for Disease Control and Prevention, Atlanta, GA) was used to create a database of epidemiological, microbiological and genotyping information. SAS software (version 9.1; SAS Institute) was used to perform the statistical analyses. Prevalence of pneumococci carriage and $95 \%$ confidence intervals were adjusted for the household sampling design effect. Fisher's exact test or $\chi^{2}$ test was used to evaluate significant associations for categorical data. The two-sample Wilcoxon rank-sum test was used to compare differences for continuous variables. Logistic regression modeling was performed with variables of age, sex and those that had a two-sided $P$ value $<0.05$ in univariate analyses. A backward elimination method was used to obtain a final model. Generalized estimating equations were used to obtain odds ratios (OR) and $95 \% \mathrm{CI}$ which were adjusted to account for household sampling design effect. Associations with a two-sided $P$ value $<0.05$ were defined as being statistically significant. In order to determine whether carriage strains were related to strains that caused invasive disease in Salvador, Brazil, sequence types for carriage strains were compared to those from 362 isolates obtained during active surveillance study for pneumococcal meningitis in the city of Salvador between 1996 and 2002 [19]. The OR for clone-specific invasiveness was calculated as described by Brueggemann et al. [7].

\section{RESULTS}

\section{Prevalence and risk factors for S. pneumoniae carriage among slum residents}

We identified 282 eligible subjects during visits of 39 households. The mean number of inhabitants per households was 7 (range: 3 to 14 ). Mean monthly per capita household income was US\$ 73.00 (inter-quartile range, 39.8-98.4). Among 282 eligible subjects, 262 (93\%) were enrolled for the survey, of which 57\% were female and median age was 16 years. Among the 39 study households, $14(36 \%)$ and $31(79 \%)$ had at least one child $<2$ and $<5$ years, respectively.

The prevalence of S. pneumoniae nasopharyngeal carriage was 36\% (95 of 262 subjects, $95 \%$ CI 28-44\%). Among study households, 25 (64\%) had two or more inhabitants who had pneumococcal carriage. The prevalence of carriage decreased significantly with increasing age (Figure 1). The prevalence for children $<5$ and 5-17 years of age and adults $>17$ years of age 
was $66.6 \%$ (33 of 50 subjects; $95 \%$ CI $51.2-78.8 \%$ ), $45.3 \%$ (43 of 95; 95\%CI 35-55.8\%), and $16.2 \%$ (19 of 117 ; $95 \%$ CI $10.1-24.2 \%)$, respectively.

Univariate analysis showed that age $<17$ years, school attendance and an upper respiratory tract infection (URTI) in the previous month were associated with nasopharyngeal carriage of S. pneumoniae (Table 1). Breastfeeding was associated with a decreased risk for pneumococcal carriage among children less than or equal to 2 years. Multivariate analysis identified age $<5$ years old, URTI in the previous month and school attendance as independent risk factors for pneumococcal carriage (Table 1).

\section{Antimicrobial susceptibility and serotype distribution}

Among the 95 subjects colonized, 44 (46\%), 18 (19\%) and 5 (5\%) had isolates which had decreased susceptibility to at least one, two and three antimicrobial agent classes, respectively (Table 2). Of the 95 subjects, 21 (22\%) and 9 (10\%) were colonized with oxacillin-resistant and penicillin-nonsusceptible isolates, respectively. The frequency of resistance to antibiotics other than beta-lactams was $28 \%$ for cotrimoxazole, $15 \%$ for tetracycline, $3 \%$ for chloramphenicol and $2 \%$ for erythromycin (Table 2). The 9 penicillin-nonsusceptible isolates were also resistant to cotrimoxazole. Among subjects with penicillin-nonsusceptible (PNSP) isolates, 5 (54\% of 9) were isolated from children $<5$ years old.

The distribution of serotypes for $94(99 \%)$ of 95 isolates is shown in figure 2. Evaluation of multiple colonies from the same individual found that all colonies were of the same serotype. Among the 29 serotypes identified, the most prevalent were 19F (13\%), 6A (9\%), 23F (8\%) and non-typeable ( $8 \%)$. Among the children $<5,5-17$ years of age and adults $>17$ years of age, $36 \%$ (12 of 33), 28\% (12 of 42) and 36\% (7 of 19) had serotypes contained in the seven-valent polysaccharide-protein conjugate vaccine (PCV7). Among subjects colonized with PNSP, 55\% (5 of 9) had serotypes represented in the PCV7.

\section{Clonal relationships among carriage isolates}

Combined Box fingerprinting and PFGE analysis (Figure 3) found that 41 (44\%) of the 94 isolates were distributed among 14 related patterns (Table 3). MLST found that the 14 Box/ PFGE clonal groups were distributed among 17 STs of which 11 were novel sequence types. Among the 14 Box/PFGE clonal groups, 11 had isolates with identical MLST allelic profiles and three isolates with related STs, for which the allelic profile of one isolate differed at a single locus (Table 3). In total, 53 (66\%) of the 94 isolates were found to have unrelated Box/ PFGE patterns. Analysis of 10 randomly-selected isolates with unrelated Box/PFGE found that each had a unique ST, of which 6 were novel types. Antimicrobial resistance was not associated with any specific clonal group. The 9 PNSP (Figure 3) were distributed among seven distinct STs. In total, strains from 8 (57\%) of the 14 clonal groups found in children 5-7 years of age or adults $>17$ years of age were also isolated from children $<5$ years of age (Table 3 ).

\section{Household clustering of S. pneumoniae clonal groups}

Among the 39 households, 13 (33\%) and 9 (23\%) had more than one member who was colonized with pneumococci of the same serotype and ST, respectively (Table 4). Among the 9 clonal groups which were isolated from members of the same household, six were also found among subjects residing in other households. In 5 (13\%) of the 39 households, children 5-17 years of age and adults $>17$ years were colonized with strains with the same ST as those found in young children $<5$ years of age from the same households. 


\section{Genotypic relationship between carriage and meningitis isolates}

Genotyping of carriage isolates obtained from individuals in the slum community and patients with pneumococcal meningitis found that 19 (20\% of 94) carriage isolates had identical or closely-related PFGE patterns to those from strains isolated from meningitis cases (Figure 3). Of these, 17 carriage isolates belonged to 6 of the 14 clonal groups whereas two isolates were among the 53 carriage strains which did not belong to a clonally-related group. The 6 clonal groups represented in both carriage and meningitis isolates accounted for 26\% (94 of 362) of the cases of pneumococcal meningitis identified during surveillance. S. pneumoniae from the serotype 18C/21 ST 193 clonal group were isolated from 6\% (6 of 94) of colonized subjects and 6\% (22 of 362) of meningitis cases (OR for clone-specific invasiveness $0.95,95 \% \mathrm{CI}$ 0.35-2.70). Serotype 3 ST180 and ST1024 clonal groups were recovered from 1\% (1 of 94 subjects) of the carriage subjects and respectively, 5 and $4 \%$ (18 and 15 cases, respectively) of the meningitis cases (OR, 95\%CI; 4.87, 0.67-99.10 and 4.02, 0.55-82.65, respectively). However, the odds ratio for serotype 3 -specific invasiveness was 5.71, 95\%CI 1.32-34.85 (11\% of meningitis subjects vs. $2 \%$ of carriage subjects).

The international serotype 14 ST 66 clone was isolated from $2 \%$ (2 of 94) of the carriage subjects and 9\% (33 cases) of the meningitis cases (OR 4.61, 95\%CI 1.05-28.35). Furthermore, this clone accounted for $33(55.9 \%)$ of the 59 cases of penicillin-nonsusceptible pneumococcal meningitis during the study period. As found with serotype 3 , serotype 14 was strongly associated with the risk for acquiring invasive disease (OR, 8.78; 95\% CI 2.05-52.97; $16 \%$ of meningitis subjects vs. $2 \%$ of carriage subjects). The other ST types (ST2257, ST2259, ST776, ST2258) accounted for 12\% (11 of 94) of the carriage isolates and 2.8\% (10 of 362) of the meningitis cases (OR $0.21,95 \% \mathrm{CI} 0.08-0.56$ ).

\section{DISCUSSION}

This prevalence study performed in an urban slum community in Brazil found that $36 \%$ of the residents were carriers of S. pneumoniae. Pneumococcal carriage rates vary significantly depending on the geographic region and underlying socio-economic conditions [20-22]. However in this study, the high prevalence of pneumococcal carriage among children $<5$ years old (65\%) was similar to those reported in developing countries [23-25]. School attendance was found to be an independent predictor for carriage, suggesting that the school setting may have a role in transmission in slum communities. Although slum residents live in crowded household conditions, we did not find the number of household contacts to be a risk factor for pneumococci carriage.

Significant geographical variations in the frequency of antibiotic resistance have been observed in different countries and within the same country. Penicillin-nonsusceptible isolates were less frequent in our study population (9\%) than in the other regions of Brazil, such as northeast (41\%) and Central region (19\%) [25,26]. However, among children $<5$ years old, $12 \%$ of pneumococci were intermediately resistant, while $36 \%$ of the isolates were resistant to cotrimoxazole. Cotrimoxazole is recommended for first-line outpatient treatment of community-acquired pneumonia in developing countries [27]. In Brazil the widespread use of this commonly prescribed antimicrobial agent in the outpatient setting has presumably provided the selection pressure for cotrimoxazole resistant strains. Of note, $100 \%$ of the penicillin-nonsusceptible carriage isolates were nonsusceptible to cotrimoxazole, suggesting the potential for selection of penicillin-nonsusceptible strains with widespread community use of cotrimoxazole.

As expected we found that the serotype distribution among nasopharyngeal isolates from the cross-sectional study (19F, 6A, NT, 23F, 18C and 34) was significantly different from those commonly found among invasive disease isolates both in Salvador [28], as well as other regions 
in Brazil [26]. Among carriage isolates from children $<5$ years of age, $36 \%$ had serotypes represented in the PCV7 whereas 74\% of the invasive isolates from children of the same age group had vaccine serotypes [28]. The contribution of PCV7 serotypes in pneumococcal carriage varies between geographical regions [29-31]. However, the proportion of carriage isolates with PCV7 serotypes in children appears to be substantially lower than generally observed in industrialized countries [20,29,32] Among PNSP from all ages, 44\% were NVT, highlighting the potential of serotypes which are not covered by PCV7 to develop penicillin resistance. Together these findings may have important implications if conjugate vaccines will be implemented in similar urban populations in the future.

Several studies have systematically explored the transmission of pneumococci within households and communities [29,33-36]. These studies, although performed in distinct geographic regions, found that $S$. pneumoniae transmission within families occurs from children to adults $[29,33,37]$. We did not observe that prevalence of carriage within households was associated with the size of the household or numbers of contacts, which would suggest a density-dependent within-household transmission phenomenon [35], albeit the study's crosssectional design and sample size may have limited the ability to adequately evaluate this possibility. Attendance in child care centers is an important risk factor for pneumococcal carriage in industrialized countries and may explain the variations in carriage observed between communities in this setting [8,30,38]. In impoverished slum communities such as the study population, access to child care centers is often limited or non-existent. However, we did find that school attendance was an independent risk factor for pneumococcal carriage, suggesting that these sites may be potentially important sources for transmission in slum communities.

Few studies have investigated the community transmission of pneumococci at level of clonal composition $[8,39]$. In Israel, a study conducted in a closed community (a kibbutz) found that strains carried by day care attendees were not isolated either from their parents or from other adult members of the community [39]. This is the first study in an open community - i.e., enrolling child and adult family members as well as other individuals living in the vicinity. In the community site where the study was performed, families have similar standards of living and the relationship between carriage of pneumococci by young children and that found among families and members of the community could be readily investigated.

In this community-based study, $43 \%$ of the nasopharyngeal pneumococcal isolates belonged to clonally-related groups. This finding suggests that there is enhanced spread of pneumococci between households in the slum community. Moreover, we found that $11.9 \%$ and $31.6 \%$ of the older children and adolescents (5-17 years of age) and adults, respectively were colonized with the same clonal groups of strains carried by the younger children in the same household. Intra-household spread of S. pneumoniae may therefore be an important mode of transmission in this epidemiological setting.

We found that within the community site, the survey of nasopharyngeal carriage isolates of $S$. pneumoniae reflects the antibiotic susceptibility pattern of meningitis isolates found in the same time period for most antibiotics. However the serotype distribution and molecular analysis of PNSP showed that carriage isolates are more diverse and represent distinct clonal groups, most of which were not identified during meningitis disease surveillance. Studies of isolates from patients with invasive disease in Brazil $[11,12]$ found that a high proportion of PNSP isolates are restricted to a few clonal groups, of which ST66 is predominantly represented [12]. In this study, ST66 accounted for $22 \%$ of the penicillin-nonsusceptible carriage isolates whereas it was the cause of $56 \%$ of the cases of PNSP meningitis in the city during the same time period. 
As observed in other studies which have compared carriage and invasive isolates [6-8], different clonal groups identified in this study had different potentials for causing invasive disease. Among major clonal groups isolated from meningitis patients, the serotype 14 ST66 clone had significantly increased potential (OR 4.61, 95\% CI 1.05-28.33) for causing invasive disease. This association was not observed for the other 7 STs which were found in both carriage and meningitis isolates. Although serotype 3 clones (ST180 and ST1024) and serotype 18C/ 21 clones (ST193/2981) did not demonstrate significantly increased invasiveness, the presence of serotype 3 capsule was associated with increased invasiveness (OR 5.71, 95\% CI 1.32-34.85), suggesting that this phenotype, independent of or in addition to genotype, may be a factor in promoting disease invasiveness after colonization. These findings support those reported previously that invasive potential of the isolate may be related to its capsular [7], yet the small numbers of isolates in our study limited the ability to evaluate significant independent inferences.

In contrast to findings from other studies [6-8] our findings suggest that in this study community, the majority of carriage genotypes may not cause invasive disease. Among carriage isolates, $78 \%$ had genotype patterns not found among meningitis isolates obtained during the surveillance period. A caveat in interpreting these results is the small number of carriage isolates and collection of isolates at a single time-point. Furthermore, the nasopharyngeal carriage study was conducted in one of a large number of slum communities in the city. However, the study community has a similar demographic and socio-economic profile to other slum communities in Salvador and Brazilian urban centers, indicating that the study's findings may be generalizable to these situations. The study was also limited in that comparisons were made with isolates that were obtained from cases with meningitis and did not include other forms of invasive pneumococcal disease. Finally, it was difficult to address the question of whether differences in the invasive potential relates to the serotype or clonespecific properties of pneumococcal strains due to the limited number of clonal groups within a serotype.

Major initiatives are being made by governments through support from PneumoADIP and the GAVI Alliance to extend the public health benefits afforded by PCV7 to developing countries [10]. A large proportion of the population in these countries resides in slum settlements, and the world's slum population is expected to double within the next 20 years [9]. Serotype shift and replacement, together with clonal expansion of pneumococci with non-vaccine serotypes, have become major considerations with the use of polysaccharide conjugate vaccine [40]. Our study's findings indicate that $S$. pneumoniae spread efficiently in slum settings as evidenced by the carriage of related clones within and between households. This could thereby facilitate clonal dissemination of non-vaccine serotypes if conjugate vaccines were to be implemented in these communities. In developed countries where conjugate vaccines have been used, immunization with PCV7 effectively reduces carriage from S. pneumoniae with vaccine serotypes [37,38], yet studies, have documented the increase in carriage and invasive disease of some non-vaccine serotypes [30,38,41]. The importance of non-vaccine serotypes circulating in developing countries and their ability to cause invasive disease is still not clear.

Further research is therefore needed to determine whether the repertoire of non-vaccine serotypes and clones associated with carriage in this study have invasive disease potential. This will be an important consideration for the eventual introduction of conjugate vaccines in slum communities in developing countries.

\section{ACKNOWLEDGMENTS}

We thank the families of the study community which participated in the study. We also thank laboratory assistants Cíntia Carla, Adriano Queiroz and Maviany Mota, for their immensurable help; Richard Facklam for advice during laboratory analysis and confirmation of the serotyping results. This work was supported by grants from the Oswaldo 
Cruz Foundation, Brazilian Ministry of Health (0250.250.415); the Brazilian National Research Council (300.861/96-6, 521.132/98-3 and PRONEX 4196086200); the Research Foundation for the State of Bahia (1431040054051) and the National Institutes of Health (D43 TW00919 and R01 TW007303).

This work was supported by grants from the Oswaldo Cruz Foundation, Brazilian Ministry of Health (0250.250.415); the Brazilian National Research Council (300.861/96-6, 521.132/98-3 and PRONEX 4196086200); the Research Foundation for the State of Bahia (1431040054051) and the National Institutes of Health (D43 TW00919 and R01 TW007303).

\section{REFERENCES}

1. Giebink GS. The prevention of pneumococcal disease in children. N Engl J Med 2001;345:1177-83. [PubMed: 11642234]

2. Faden H, Duffy L, Wasielewski R, Wolf J, Krystofik D, Tung Y. Relationship between nasopharyngeal colonization and the development of otitis media in children. Tonawanda/Williamsville Pediatrics. J Infect Dis 1997;175:1440-5. [PubMed: 9180184]

3. Bogaert D, De Groot R, Hermans PW. Streptococcus pneumoniae colonization: the key to pneumococcal disease. Lancet Infect Dis 2004;4:144-54. [PubMed: 14998500]

4. de Andrade AL, Pimenta FC, Brandileone MC, et al. Genetic relationship between Streptococcus pneumoniae isolates from nasopharyngeal and cerebrospinal fluid of two infants with Pneumococcal Meningitis. J Clin Microbiol 2003;41:3970-2. [PubMed: 12904432]

5. Takala AK, Vuopio-Varkila J, Tarkka E, Leinonen M, Musser JM. Subtyping of common pediatric pneumococcal serotypes from invasive disease and pharyngeal carriage in Finland. J Infect Dis 1996;173:128-35. [PubMed: 8537649]

6. Robinson DA, Edwards KM, Waites KB, Briles DE, Crain MJ, Hollingshead SK. Clones of Streptococcus pneumoniae isolated from nasopharyngeal carriage and invasive disease in young children in central Tennessee. J Infect Dis 2001;183:1501-7. [PubMed: 11319686]

7. Brueggemann AB, Griffiths DT, Meats E, Peto T, Crook DW, Spratt BG. Clonal relationships between invasive and carriage Streptococcus pneumoniae and serotype- and clone-specific differences in invasive disease potential. J Infect Dis 2003;187:1424-32. [PubMed: 12717624]

8. Sandgren A, Sjostrom K, Olsson-Liljequist B, et al. Effect of clonal and serotype-specific properties on the invasive capacity of Streptococcus pneumoniae. J Infect Dis 2004;189:785-96. [PubMed: 14976594]

9. Programme UNHS. The challenge of slums: global report on human settlements. London and Sterling: Earthscan Publications Ltd.; London: 2003. p. 310

10. Sinha A, Levine O, Knoll MD, Muhib F, Lieu TA. Cost-effectiveness of pneumococcal conjugate vaccination in the prevention of child mortality: an international economic analysis. Lancet 2007;369:389-96. [PubMed: 17276779]

11. Ko AI, Reis JN, Coppola SJ, et al. Clonally related penicillin-nonsusceptible Streptococcus pneumoniae serotype 14 from cases of meningitis in Salvador, Brazil. Clin Infect Dis 2000;30:7886. [PubMed: 10619737]

12. Brandileone MC, Di Fabio JL, Vieira VS, et al. Geographic distribution of penicillin resistance of Streptococcus pneumoniae in Brazil: genetic relatedness. Microb Drug Resist 1998;4:209-17. [PubMed: 9818972]

13. Secretaria de combate à pobreza e às desigualdades sociais/SECOMP: Mapeamento da pobreza em áreas urbanas do estado da Bahia. 2005. CD-ROM

14. IBGE. Anuário Estatístico do Brasil. Vol. 56. Instituto Brasileiro de Geografia e Estatística; Rio de Janeiro: 2000.

15. CLSI. Clinical and Laboratory Standards institute (formely NCCLS): Performance standards for antimicrobial susceeptibility testing, 12th informational supplement. PA NCCLS; Wayne: 2002.

16. Lefevre JC, Faucon G, Sicard AM, Gasc AM. DNA fingerprinting of Streptococcus pneumoniae strains by pulsed-field gel electrophoresis. J Clin Microbiol 1993;31:2724-8. [PubMed: 8253973]

17. Tenover FC, Arbeit RD, Goering RV, et al. Interpreting chromosomal DNA restriction patterns produced by pulsed-field gel electrophoresis: criteria for bacterial strain typing. J Clin Microbiol 1995;33:2233-9. [PubMed: 7494007] 
18. Enright MC, Spratt BG. A multilocus sequence typing scheme for Streptococcus pneumoniae: identification of clones associated with serious invasive disease. Microbiology 1998;144(Pt 11): 3049-60. [PubMed: 9846740]

19. Reis, JN.; Cordeiro, SM.; Gouveia, EL., et al. Molecular Epidemiology of Pneumococcal Meningitis during Seven-Year Surveillance in Salvador, Brazil. 103rd General Meeting; Washington, D.C.: American Society for Microbiology; 2003.

20. Bogaert D, Sluijter M, Toom NL, et al. Dynamics of pneumococcal colonization in healthy Dutch children. Microbiology 2006;152:377-85. [PubMed: 16436426]

21. Feikin DR, Davis M, Nwanyanwu OC, et al. Antibiotic resistance and serotype distribution of Streptococcus pneumoniae colonizing rural Malawian children. Pediatr Infect Dis J 2003;22:564-7. [PubMed: 12828156]

22. Bayraktar MR, Durmaz B, Kalcioglu MT, Durmaz R, Cizmeci Z, Aktas E. Nasopharyngeal carriage, antimicrobial susceptibility, serotype distribution and clonal relatedness of Streptococcus pneumoniae isolates in healthy children in Malatya, Turkey. Int J Antimicrob Agents 2005;26:2416. [PubMed: 16118046]

23. Levine S, Dejsirilert S, Sangsuk L, et al. Serotypes and antimicrobial resistance of Streptococcus pneumoniae in Thailand 2002-2004. Pediatr Infect Dis J 2006;25:176-8. [PubMed: 16462300]

24. Denno DM, Frimpong E, Gregory M, Steele RW. Nasopharyngeal carriage and susceptibility patterns of Streptococcus pneumoniae in Kumasi, Ghana. West Afr J Med 2002;21:233-6. [PubMed: 12744576]

25. Rey LC, Wolf B, Moreira JLB, Verhoef J, Farhat CK. S. pneumoniae isolados da nasofaringe de crianças sadias e com pneumonia: taxa de colonização e susceptibilidade aos antimicrobianos. Jornal de Pediatria 2002;78:105-12. [PubMed: 14647791]

26. Laval CB, de Andrade AL, Pimenta FC, et al. Serotypes of carriage and invasive isolates of Streptococcus pneumoniae in Brazilian children in the era of pneumococcal vaccines. Clin Microbiol Infect 2006;12:50-5. [PubMed: 16460546]

27. Who. Recommendation for first-line outpatient treatment of community acquired pneumonia in developing countries. 2002.

28. Reis JN, Cordeiro SM, Coppola SJ, et al. Population-based survey of antimicrobial susceptibility and serotype distribution of Streptococcus pneumoniae from meningitis patients in Salvador, Brazil. J Clin Microbiol 2002;40:275-7. [PubMed: 11773131]

29. Hussain M, Melegaro A, Pebody RG, et al. A longitudinal household study of Streptococcus pneumoniae nasopharyngeal carriage in a UK setting. Epidemiol Infect 2005;133:891-8. [PubMed: 16181510]

30. Huang SS, Platt R, Rifas-Shiman SL, Pelton SI, Goldmann D, Finkelstein JA. Post-PCV7 changes in colonizing pneumococcal serotypes in 16 Massachusetts communities, 2001 and 2004. Pediatrics 2005;116:e408-13. [PubMed: 16140686]

31. Hill PC, Akisanya A, Sankareh K, et al. Nasopharyngeal carriage of Streptococcus pneumoniae in Gambian villagers. Clin Infect Dis 2006;43:673-9. [PubMed: 16912937]

32. Melegaro A, Choi Y, Pebody R, Gay N. Pneumococcal carriage in United Kingdom families: estimating serotype-specific transmission parameters from longitudinal data. Am J Epidemiol 2007;166:228-35. [PubMed: 17517684]

33. Shimada J, Yamanaka N, Hotomi M, et al. Household transmission of Streptococcus pneumoniae among siblings with acute otitis media. J Clin Microbiol 2002;40:1851-3. [PubMed: 11980976]

34. Hoshino K, Watanabe H, Sugita R, et al. High Rate of Transmission of Penicillin-Resistant Streptococcus pneumoniae between Parents and Children. Journal of Clinical Microbiology 2002;40:4357-59. [PubMed: 12409431]

35. Melegaro A, Gay NJ, Medley GF. Estimating the transmission parameters of pneumococcal carriage in households. Epidemiol Infect 2004;132:433-41. [PubMed: 15188713]

36. Huang SS, Finkelstein JA, Lipsitch M. Modeling community- and individual-level effects of childcare center attendance on pneumococcal carriage. Clin Infect Dis 2005;40:1215-22. [PubMed: 15825020] 
37. Whitney CG, Farley MM, Hadler J, et al. Decline in invasive pneumococcal disease after the introduction of protein-polysaccharide conjugate vaccine. N Engl J Med 2003;348:1737-46. [PubMed: 12724479]

38. Dagan R, Givon-Lavi N, Zamir O, Fraser D. Effect of a nonavalent conjugate vaccine on carriage of antibiotic-resistant Streptococcus pneumoniae in day-care centers. Pediatr Infect Dis J 2003;22:53240. [PubMed: 12799510]

39. Borer A, Meirson H, Peled N, et al. Antibiotic-Resistant Pneumococci Carried by Young Children Do Not Appear to Disseminate to Adult Members of a Closed Community. Clinical Infectious Disease 2001;33:436-44.

40. Singh NK. Pneumococcal vaccine concerns: shape-shifters and beyond. Vaccine 2007;25:5244-5. [PubMed: 17566614]

41. Frazao N, Brito-Avo A, Simas C, et al. Effect of the seven-valent conjugate pneumococcal vaccine on carriage and drug resistance of Streptococcus pneumoniae in healthy children attending day-care centers in Lisbon. Pediatr Infect Dis J 2005;24:243-52. [PubMed: 15750461] 


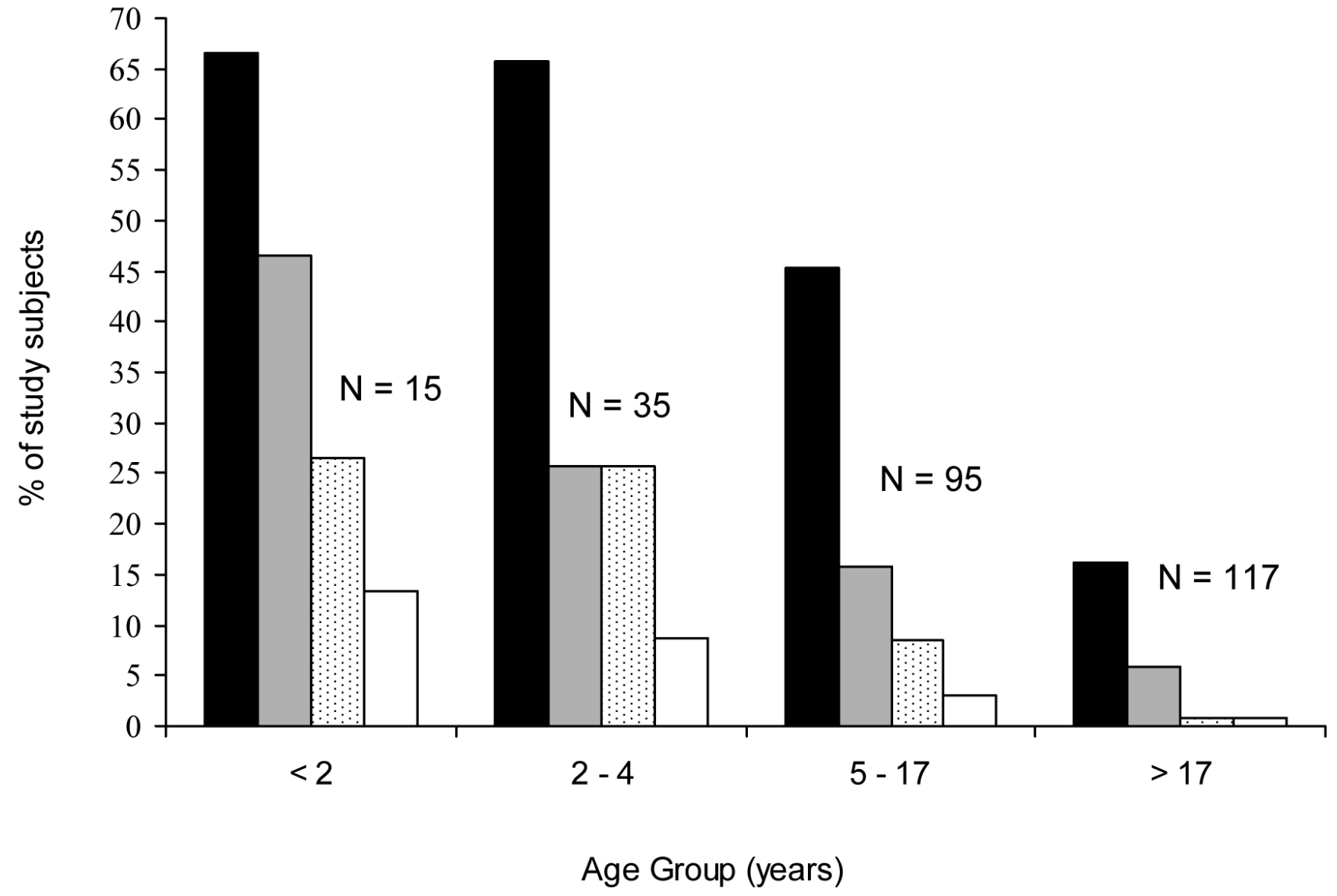

Figure 1.

Proportion of slum residents ( $\mathrm{N}=262)$ with nasopharyngeal carriage of S. pneumoniae, according to age group and antimicrobial susceptibility. Proportion are shown for individuals colonized with pneumococci (black bars), colonized with cotrimoxazole non-susceptible isolates (gray bars), colonized with oxacillin resistant isolates (dotted bars), and colonized with penicillin-nonsusceptible isolates (white bars). 


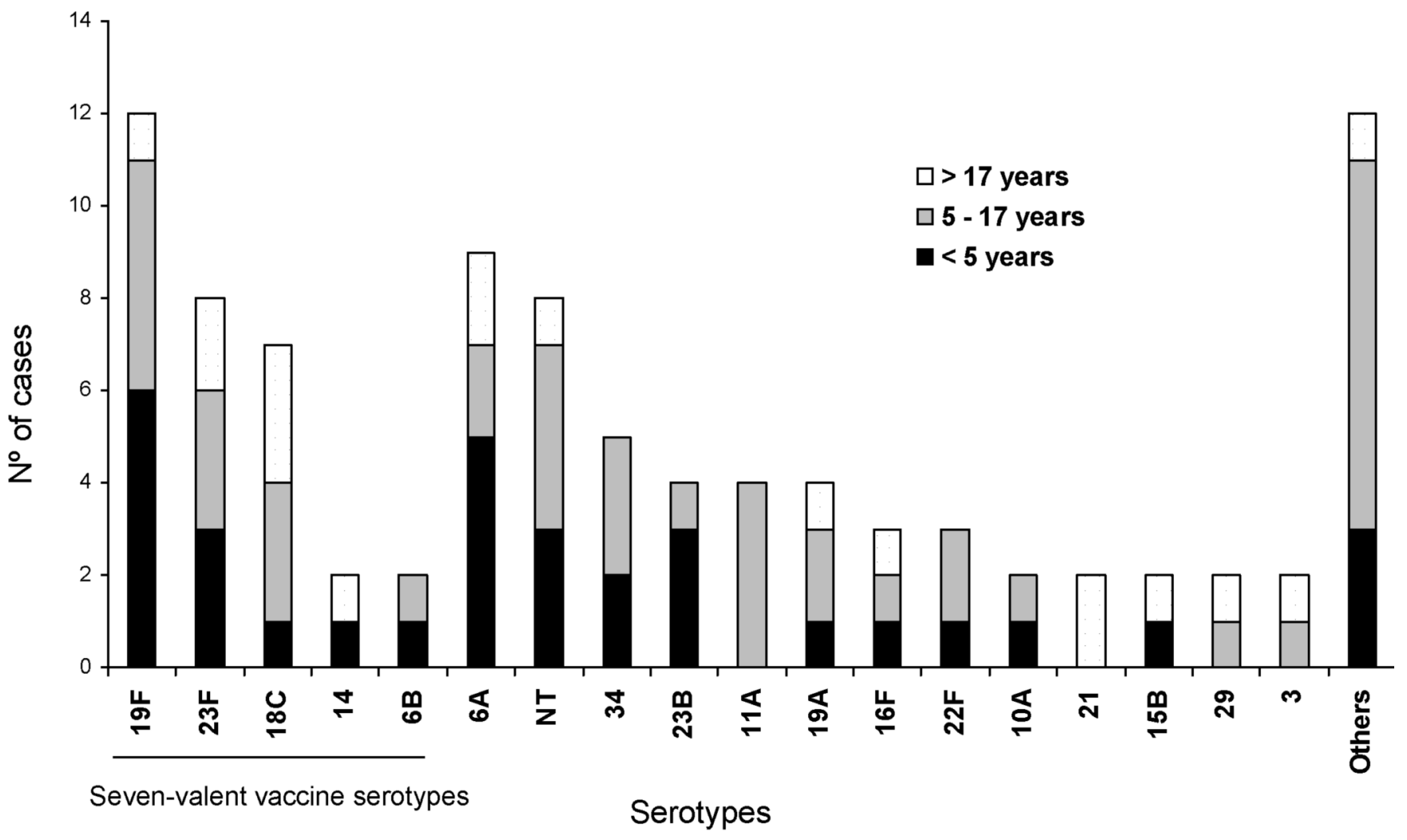

Figure 2.

Serotype distribution of 94 nasopharyngeal carriage Streptococcus pneumoniae isolates. 


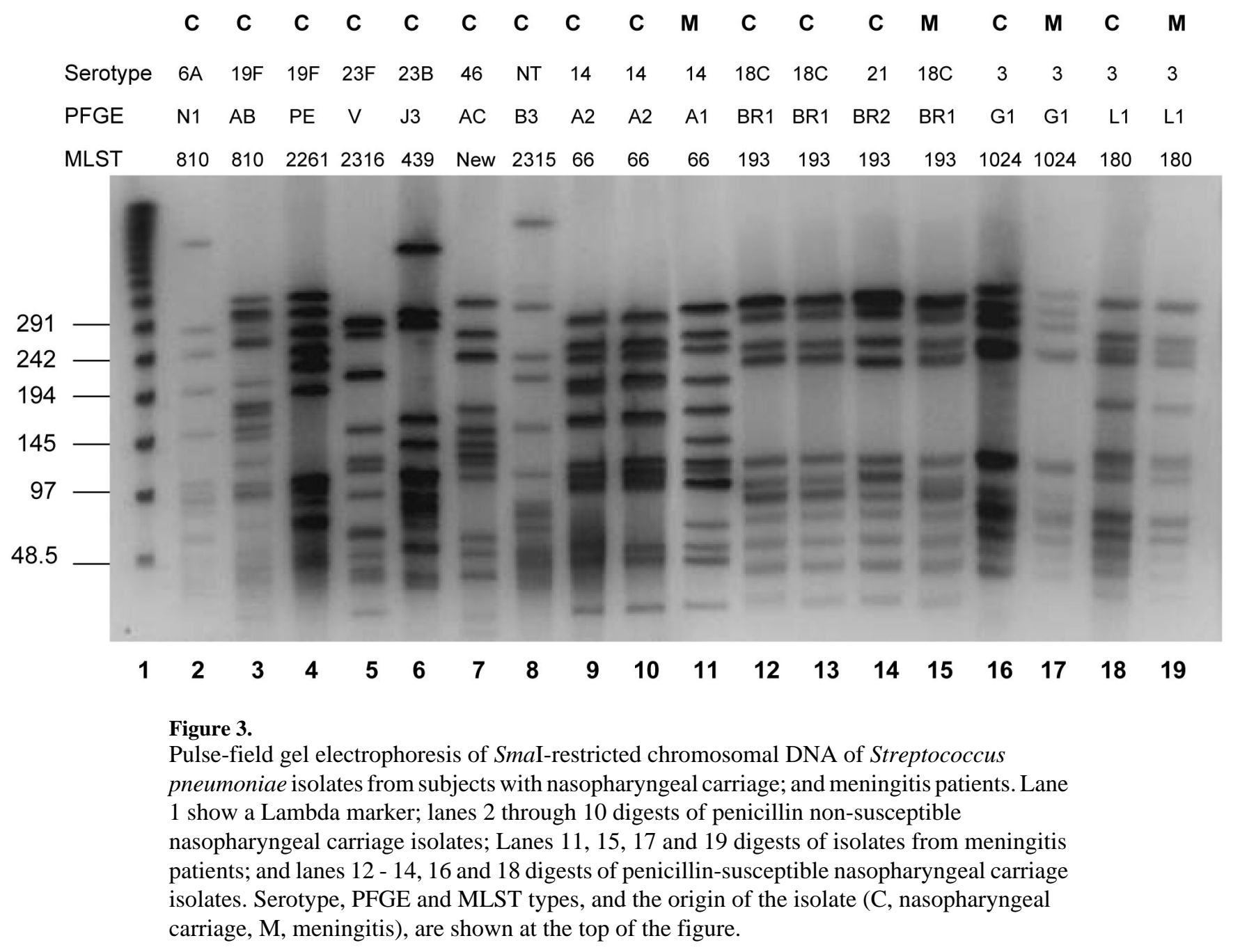

J Infect. Author manuscript; available in PMC 2009 September 1. 
Table 1

Univariate and multivariate analysis of risk factors for pneumococcal carriage among urban slum residents.

\begin{tabular}{|c|c|c|c|c|c|}
\hline \multirow{2}{*}{\multicolumn{2}{|c|}{ Characteristics }} & \multicolumn{2}{|c|}{ Pneumococcal carriage } & \multirow[t]{2}{*}{ Univariate analysis } & \multirow[t]{2}{*}{ Multivariate analysis $^{a}$} \\
\hline & & $\operatorname{Yes}(\mathrm{N}=95)$ & No $(N=167)$ & & \\
\hline & & & & & CI) \\
\hline \multirow[t]{4}{*}{ Age } & $<2 \mathrm{yrs}$ & $10(11)$ & $5(3)$ & $7.9(2.2-28.7)$ & $14.1(5.2-38.2)$ \\
\hline & $2-4 \mathrm{yrs}$ & $23(24)$ & $12(7)$ & $8.9(4.1-19.4)$ & $8.0(3.5-18.6)$ \\
\hline & $5-17 \mathrm{yrs}$ & $43(45)$ & $52(31)$ & $4.7(2.6-8.7)$ & $1.5(0.6-3.8)$ \\
\hline & $>17 \mathrm{yrs}$ & $19(20)$ & $98(59)$ & 1.0 & 1.0 \\
\hline \multicolumn{2}{|l|}{ Male gender } & $48(51)$ & $65(39)$ & $1.4(1.0-2.0)$ & $1.1(0.7-1.8)$ \\
\hline \multicolumn{2}{|c|}{ Chronic underlying illness } & $13(14)$ & $31(19)$ & $0.7(0.3-1.4)$ & \\
\hline \multicolumn{2}{|c|}{ URTI in the last month } & $40(42)$ & $38(23)$ & $2.1(1.3-3.6)$ & $1.6(0.9-2.8)$ \\
\hline \multicolumn{2}{|c|}{ Antibiotic use in the last month } & $11(12)$ & $15(9)$ & $1.1(0.5-2.6)$ & \\
\hline \multicolumn{2}{|l|}{ Breastfeeding } & $4(4)$ & $4(2)$ & $0.1(0.02-0.7)$ & $0.2(0.05-1.0)$ \\
\hline \multicolumn{2}{|l|}{ Day care attendance } & $8(8)$ & 2(1) & $1.3(0.3-5.3)$ & \\
\hline \multicolumn{2}{|l|}{ School attendance } & $39(41)$ & $41(24)$ & $2.1(1.4-3.0)$ & $2.7(1.2-6.0)$ \\
\hline \multicolumn{2}{|l|}{ Smoking } & $4(4)$ & $17(10)$ & $0.6(0.2-1.5)$ & \\
\hline \multicolumn{2}{|c|}{ Household contact who is a smoker } & $43(45)$ & $69(41)$ & $1.1(0.5-2.3)$ & \\
\hline \multirow{3}{*}{$\begin{array}{l}\text { No. of household } \\
\text { contacts }\end{array}$} & $<5$ & $7(7)$ & $14(8)$ & 1.0 & \\
\hline & $5-10$ & $58(61)$ & 99 (59) & $1.1(0.5-2.6)$ & \\
\hline & $>10$ & $30(32)$ & $54(32)$ & $1.0(0.4-2.7)$ & \\
\hline \multirow{2}{*}{$\begin{array}{l}\text { Household } \\
\text { contact with age }\end{array}$} & $<5 \mathrm{yrs}$ & $69(73)$ & 129 (77) & $0.7(0.3-1.4)$ & \\
\hline & $<10 \mathrm{yrs}$ & 89 (94) & 149 (89) & $1.7(0.5-4.6)$ & \\
\hline \multicolumn{2}{|l|}{$\begin{array}{l}\text { Household } \\
\text { contact with } \\
\text { pneumococcal } \\
\text { carriage }\end{array}$} & $86(91)$ & $148(89)$ & $1.2(0.5-3.1)$ & \\
\hline
\end{tabular}

NOTE: URTI, upper respiratory tract infection. Odds ratio and 95\%CI adjusted for household sampling design effect and weighted for number of household members.

${ }^{a}$ Final best-fit model includes age, sex, at least one episode of URTI in the last month, breastfeeding and school attendance. 
Table 2

Antimicrobial resistance in Streptococcus pneumoniae nasopharyngeal carriage isolates.

\begin{tabular}{|c|c|c|c|c|}
\hline Antimicrobial agent ${ }^{a}$ & $\begin{array}{c}\text { Children }<5 \text { yrs }(\mathrm{n} \\
=33)\end{array}$ & $\begin{array}{c}\text { Children } 5-17 \text { yrs (n } \\
=43)\end{array}$ & $\begin{array}{c}\text { Adults }>17 \text { yrs }(n= \\
19)\end{array}$ & Total $(\mathbf{n}=95)$ \\
\hline & \multicolumn{4}{|c|}{ No (\%) of isolates } \\
\hline Oxacillin & $13(39)$ & $8(19)$ & $1(5)$ & $22(23)$ \\
\hline Penicillin $b$ & $5(15)$ & $3(7)$ & $1(5)$ & $9(9)$ \\
\hline Erythromycin & $1(3)$ & $1(2)$ & $0(0)$ & $2(2)$ \\
\hline Tetracycline & $8(24)$ & $4(9)$ & $2(10)$ & $14(15)$ \\
\hline Chloramphenicol & $2(6)$ & $0(0)$ & $1(5)$ & $3(3)$ \\
\hline Cotrimoxazole & 16 & 15 & 7 & 38 \\
\hline Intermediate & $4(12)$ & $5(12)$ & $2(10)$ & $11(11)$ \\
\hline Resistant & $12(36)$ & $10(23)$ & $5(26)$ & $27(28)$ \\
\hline$\geq 2$ agents $c$ & $11(33)$ & $4(9)$ & $3(16)$ & $18(19)$ \\
\hline$\geq 3$ agents $^{d}$ & $3(9)$ & $0(0)$ & $2(10)$ & $5(5)$ \\
\hline
\end{tabular}

NOTE. Breakpoints $(\mu \mathrm{g} / \mathrm{ml})$ used to define susceptibility categories were those recommended by the Clinical and Laboratory Standards Institute (5). Disk diffusion method was used to determine susceptibility to all drugs tested except to penicillin.

$a_{\text {Includes intermediate and resistant strains. }}$

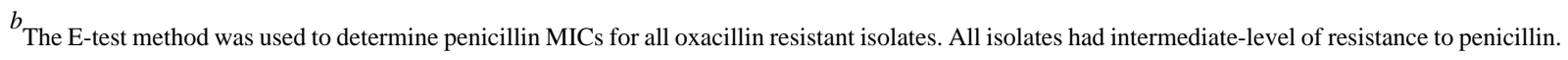

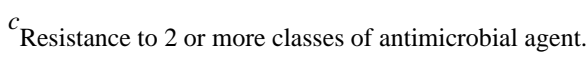

$d_{\text {Resistance to } 3 \text { or more classes of antimicrobial agent. }}$ 


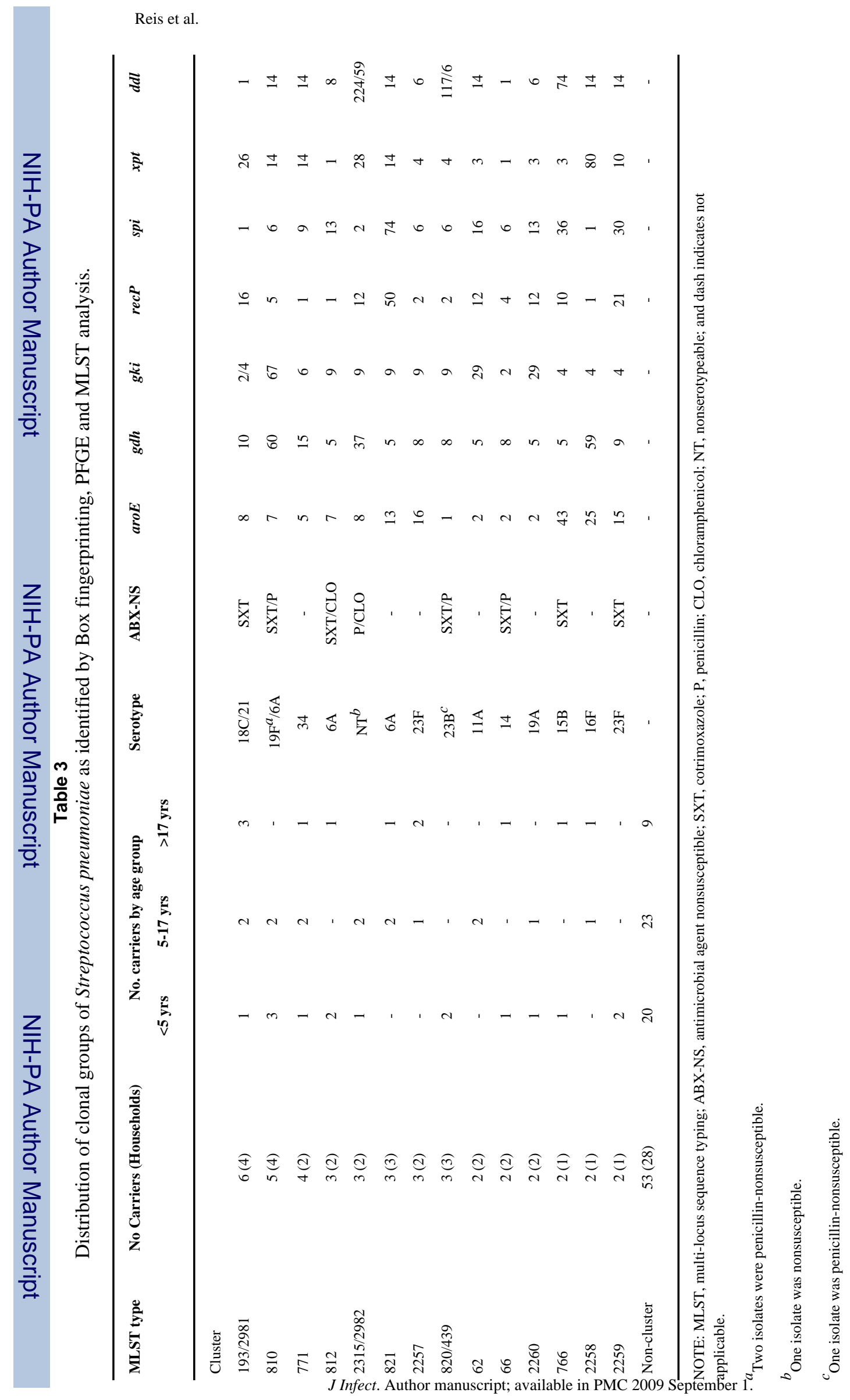


Table 4

Distribution of clonal groups of Streptococcus pneumoniae nasopharyngeal isolates among children and adults from the same household.

\begin{tabular}{|c|c|c|c|c|c|c|}
\hline \multicolumn{7}{|c|}{ No. carriers by age group } \\
\hline Household No. & No. of residents & $>5 \mathrm{yrs}$ & $5-17 y r s$ & $<17 \mathrm{yrs}$ & Serotype (ABX-NS) & MLST type \\
\hline 2 & 11 & 1 & 1 & 1 & 34 & 771 \\
\hline 4 & 8 & 1 & - & 1 & $15 \mathrm{~B}(\mathrm{SXT})$ & 766 \\
\hline 6 & 14 & 1 & - & 1 & $18 \mathrm{C}(\mathrm{SXT})$ & 193 \\
\hline 19 & 7 & - & 1 & 1 & $23 \mathrm{~F}$ & 2257 \\
\hline 22 & 14 & 1 & 1 & - & NT & 2315 \\
\hline 29 & 8 & - & 1 & 1 & $16 \mathrm{~F}$ & 2258 \\
\hline 30 & 10 & 2 & - & 1 & 6A (SXT/CLO) & 812 \\
\hline 38 & 12 & 1 & 1 & - & $19 \mathrm{~F}(\mathrm{SXT})$ & 810 \\
\hline 39 & 3 & 2 & - & - & $23 \mathrm{~F}(\mathrm{SXT})$ & 2259 \\
\hline
\end{tabular}

NOTE: ABX-NS, antimicrobial agent non-susceptible; CLO, chloramphenicol; SXT, cotrimoxazole; NT, nonserotypeable. 